\title{
Probiotics to Prevent Clostridium difficile Infection in Patients Receiving Antibiotics
}

\author{
Joshua Z. Goldenberg, ND; Dominik Mertz, MD; Bradley C. Johnston, PhD
}

CLINICAL QUESTION In adults and children prescribed antibiotics, is co-administration of a probiotic associated with a lower risk of symptomatic Clostridium difficile infection without an increase in adverse events?

воттOM LINE Moderate-quality evidence suggests that probiotics are associated with a lower risk of $C$ difficile infection and very low-quality evidence suggests that probiotics are associated with fewer adverse events vs placebo or no treatment.

Introduction

Antibiotics are commonly prescribed and are associated with Clostridium difficile infection (CDI) and its complications including toxic megacolon and death. ${ }^{1}$ Recent data suggest that treating CDI costs $\$ 24205$ per patient. ${ }^{2}$ This JAMA Clinical Evidence Synopsis summarizes a recent Cochrane review. ${ }^{3}$

\section{Summary of Findings}

Co-administration of probiotics and antibiotics was associated with a lower risk for CDI vs placebo or no treatment (70/4525 [1.5\%] vs 164/ 4147 [4.0\%], respectively; risk ratio [RR], 0.40 [95\% Cl, 0.30-0.52], $P<.001$ ) based on moderate-quality evidence using the Grading of Recommendations Assessment, Development and Evaluation (GRADE) and number needed to treat (NNT) of 40 (Figure). The results did not substantially change in predefined subgroups consisting of inpatients, outpatients, adults, children, probiotic species or strains, lower probiotic doses, higher probiotic doses, or studies at high risk vs low risk of bias.

In a post hoc subgroup analysis of trials grouped by baseline risk of CDI (determined by the event risk for placebo or no treatment), there was no association of probiotics plus antibiotics with CDI reduction in trials with a risk of CDI of $0 \%-2 \%$ or $3 \%-5 \%$ vs placebo or no treatment; however, there was an association with CDI reduction in trials with CDI risk greater than 5\% (43/1370 [3.1\%] vs 126/1084 [11.6\%]; RR, 0.30 [95\% Cl, 0.21-0.42], $P=.01$; GRADE: moderatequality evidence; NNT, 12).

Probiotics plus antibiotics were associated with a lower risk of adverse events such as abdominal cramping and nausea vs placebo or no treatment (620/4329 [14.3\%] vs 677/3976 [17.0\%]; RR, 0.83 [95\% Cl, 0.71 to 0.97 ], $P=.02$; GRADE: very low-quality evidence; NNT, 37). No trials reported serious adverse events attributable to probiotics. Probiotics plus antibiotics were associated with a lower risk of antibiotic-associated diarrhea vs placebo or no treatment (565/4618 [12.2\%] vs 771/4252 [18.1\%]; RR, 0.58 [95\% Cl, 0.48 to 0.70$], P<.001$; GRADE: low-quality evidence; NNT, 17).

\section{Discussion}

Among 31 studies comparing antibiotics and probiotics vs placebo or no treatment for preventing CDI in patients receiving antibiotics, probiotics were associated with a lower risk of CDI, adverse events, and antibiotic-associated diarrhea. In a post hoc analysis, a statistically significant association between probiotic use and the risk of CDI was found among trials with a high baseline CDI risk ( $>5 \%)$. However, the subgroup with a CDI risk of $3 \%$ to $5 \%$ was likely underpowered. Typical US hospitals have a CDI risk below 5\%.

\section{Limitations}

First, trials were combined across clinical settings (inpatient vs outpatient), age, probiotic strains and dose, and risk of bias. Second, trials were conducted in immunocompetent patients only, thus, generalizability to immunocompromised patients is unclear. Third, not all trials were appropriately blinded, although excluding unblinded studies did not substantially change the results.

\section{Comparison of Findings With Current Practice Guidelines}

Current guidelines from the American College of Gastroenterology, the Association for Professionals in Infection Control and Epidemiology, and the European Society of Clinical Microbiology and Infectious Diseases do not specifically recommend probiotic prophylaxis when antibiotics are prescribed, citing insufficient evidence.

\section{Evidence Profile}

No. of randomized clinical trials: 39 (31 providing data on CDI) Study years: 1988-2012 (conducted), 1989-2016 (published) Last search date: March 21, 2017

No. of patients: 9955

Male: $52 \%$; Female: $48 \%$

Race/ethnicity: Varied by country

Age, mean (range): 51 years (0-84 years)

Setting: Inpatient, outpatient, and combined inpatient and outpatient

Countries: Bulgaria, Canada, Chile, China, Finland, Germany, Italy, Japan, the Netherlands, Norway, Poland, Russia, Sweden, Turkey, United Kingdom, United States

Comparisons: Adults (33 trials) and children (6 trials) receiving antibiotics randomized to single or multistrain probiotics vs placebo or notreatment. Probiotic genera included Saccharomyces, Lactobacilli, Bifidobacteria, and Streptococci.

Primary outcomes: CDI (diarrhea with positive stool toxin or culture), adverse events

Secondary outcomes: $C$ difficile detection (or toxin in stool without diarrhea), antibiotic-associated diarrhea 
Figure. Association of Probiotics With Prevention of Clostridium difficile Infection in Patients Receiving Antibiotics

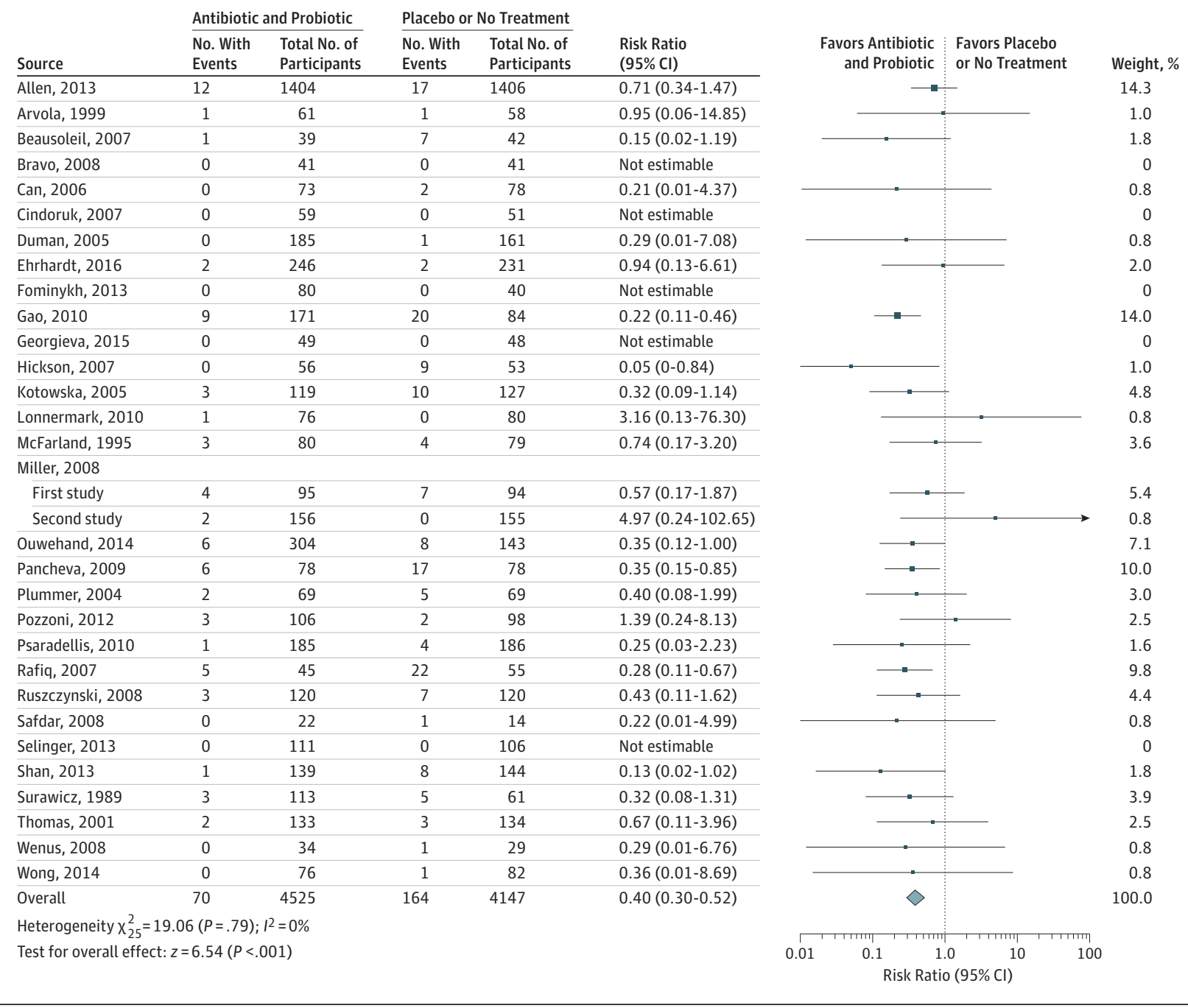

The size of the data markers is proportional to the weight of the study in the meta-analysis. The diamond represents the pooled estimate and $95 \% \mathrm{Cl}$.

Areas in Need of Future Study

Further research is needed to identify high-risk populations that may benefit most from the addition of probiotics to antibiotic treatment (eg, those known to be colonized with C difficile and those with a history of $\mathrm{CDI}$ ), and to explore the risk-to-benefit ratio in those who are immunocompromised. ${ }^{4}$

\section{ARTICLE INFORMATION}

Author Affiliations: Bastyr University Research Institute, Kenmore, Washington (Goldenberg); Australian Research Center in Complementary and Integrative Medicine, University of Technology Sydney, Ultimo (Goldenberg); Division of Infectious Diseases, Department of Medicine, McMaster University, Hamilton, Ontario, Canada (Mertz); Department of Community Health and Epidemiology, Faculty of Medicine, Dalhousie University, Halifax, Nova Scotia, Canada (Johnston).

Corresponding Author: Bradley C. Johnston, PhD Centre for Clinical Research, 5790 University Ave, Room 404, Halifax, NS B3H 1V7, Canada (bjohnston@dal.ca).

Section Editor: Mary McGrae McDermott, MD Senior Editor.
Published Online: July 19, 2018. doi:10.1001/jama.2018.9064

Conflict of Interest Disclosures: The authors have completed and submitted the ICMJE Form for Disclosure of Potential Conflicts of Interest and none were reported.

Submissions: We encourage authors to submit papers for consideration as a JAMA Clinical Evidence Synopsis. Please contact Dr McDermott at mdm608@northwestern.edu.

\section{REFERENCES}

1. Bagdasarian N, Rao K, Malani PN. Diagnosis and treatment of Clostridium difficile in adults. JAMA. 2015;313(4):398-408.
2. Nanwa N, Kendzerska T, Krahn M, et al. The economic impact of Clostridium difficile infection. Am J Gastroenterol. 2015;110(4):511-519.

3. Goldenberg JZ, Yap C, Lytvyn L, et al. Probiotics for the prevention of Clostridium difficile-associated diarrhea in adults and children. Cochrane Database Syst Rev. 2017;12:CD006095.

4. Johnston BC, Lytvyn L, Lo CK, et al. Microbial preparations (probiotics) for the prevention of Clostridium difficile infection in adults and children. Infect Control Hosp Epidemiol. 2018;39(7):771-781. 